\title{
Analysis and Development of a Localization System Based on Radio Frequency
}

\author{
D. M. Ferreira ${ }^{1}$, A. F. Ribeiro ${ }^{2}$, J.G. Rocha ${ }^{3}$, J.P. Carmo \\ ${ }^{1,2,3}$ Industrial Electronics Department, University of Minho, Campus de Azurém, 4800-058 Guimarães, Portugal \\ 11dvd.matos@gmail.com, ${ }^{2}$ fernando@dei.uminho.pt, ${ }^{3}$ gerardo@dei.uminho.pt
}

\begin{abstract}
This paper presents an analysis on several strategies for location based on Radio-Frequency. The purpose of this study is to develop an infrastructure able to determine the position of a target within an area of 0.1 acres (aprox.11000 ft2 or $1000 \mathrm{~m} 2$ ) with an acceptable precision for the specific task. Along with the analysis of the many strategies and approaches on Radio-
\end{abstract}

\section{INTRODUCTION}

As technology grows at an amazing rate, everyday we have to face numerous and various tools which, only a few years back, were only part of our imagination. Radiolocation is one of those tools, where the most commonly known system is the G.P.S. Not long ago, this technology was only available for the USA army. Nowadays, it is present in our everyday life, from airplanes to cars, from laptop computers to mobile phones. But, what if a restrictive and isolated network is required? What if the requirement is a system to use in places where the satellite signal is not available? What if the requirement is a location in a restricted area with accuracy to the centimeter, in which the G.P.S cannot guarantee security? Following is a short analysis.

Parking spaces in big metropolis are becoming a huge problem, so a frequent solution is to build parks under the ground and under buildings. It is hard to receive GPS signal on underground floors.

The largest building in the world, by volume, is the "Future of Flight Aviation Center \& Boeing Tour" with a volume of $472,000,000 \mathrm{ft}^{3}$ [1], where the 747, 746, 777 and soon the 787 [2], are built. Several persons work in this building, from engineers to technicians, janitors and caterers. To know the location of each person on the working area (for example, to know if the Master Engineer is in the assembly line area or if he is in the cafeteria) would be a tremendous tool. At the same time, Automated Guided Vehicles (or AGV) could be easily controlled and tracked down because the system would known its position on the working area, all within a restricted area, with full control of it.

For e.g.: the location of a ball in a field during a football game, its direction, speed and height, but with a precision to a point where you can determine if the ball passed the side line. FIFA has recently approved the use of microchips in soccer balls [3], in order to assist the referees in their decisions concerning the goal line. The project, proposed by Adidas, is being implemented by the company Hawk-Eye [4] which has previous experience in tennis and cricket. Although, it has proven, with good results, in the Miami Tennis Master Series, it is still an image based system. With a radio-frequency
Location, this study tries to determine the best solution to implement a working system of location in an easy, secure and less expensive way, a solution able to be used in football field, without the use of large and/or expensive infrastructures like the Global Position System (G.P.S.) network.

system, the implementation would be simpler and probably less expensive. In this case, there is a factor that could become a problem: update ratio. The system would have to be able to keep up the speed at which a ball can reach in a professional football game. In a regular game, a ball can reach up to $\left(\mathrm{v}_{\mathrm{b}}\right) 30$ $\mathrm{m} / \mathrm{s}$ (about $70 \mathrm{mph})$. If the system has a maximum error $(\Delta \mathrm{d})$ of $3 \mathrm{~cm}$, the time $\left(\mathrm{T}_{\mathrm{err}}\right)$ need to travel that distance is:

$$
T_{\text {err }}=\frac{v}{\Delta d}=\frac{30 \mathrm{~m} / \mathrm{s}}{0.03 \mathrm{~m}}=1 \mathrm{~ms}
$$

And, the update ratio would be:

$$
\text { update ratio }=\frac{1}{T_{\text {err }}}=1000 \mathrm{~Hz}
$$

Therefore, the system has to be able to update the target position, a thousand times (1000) a second, for a precision of $3 \mathrm{~cm}$, in order to be acceptably reliable.

And the possibilities must not be restricted to the ball. If a coach has a tool that records all the moves of each player on the field, he can easily create personal statistics like average speed, maximum speed, tendencies to play more on the left (or right) side, etc.

\section{STRATEGIES}

There are three fundamental strategies of radiolocation to consider:

- $\quad$ Strength of signal

- Angle of reception

- Time based

The first one, Strength of signal, is probably the easiest strategy to implement. It is used in mobile phones, cordless phones, and Bluetooth and WiFi networks. Even so, the applications using this method are as common as they are old. It is used by radio-amateurs to calibrate their transmitters, to detect interferences when implementing a wireless network infrastructure and even in Amateur Radio Direction Finding 
(ARDF), a radio guidance oriented discipline for leisure and outdoor activities for families and friends.

The principle is simple. The distance between a target (transmitter) and a receiver is related to the received signal strength. This electromagnetic signal produces a potential in the receiver's antenna, proportional to its strength. The problem is that the signal transmitted strength (power) decreases in a logarithmic proportion due to attenuation of the medium. The power loss $\left(\mathrm{P}_{\text {Loss }}\right)$ in function of the distance between the transmitter and receiver (d), depends on the power loss in an open field $\left(\mathrm{P}_{\text {Loss }, 0}\right)$ from a certain point of reference $\left(\mathrm{d}_{0}\right)$. It also depends on shadowing, a constant $\left(\mathrm{X}_{\sigma}\right)$, which is a standard deviation of a random variable described by the Gauss Law. Finally, it depends on a logarithmic function with a loss exponent (n) which varies with the environment. The system is described by:

$$
P(d)=P 0\left(d_{0}\right)+10 . n \cdot \log _{10}\left(\frac{d}{d_{0}}\right)+X_{\sigma}
$$

As such, it would be necessary to use strong amplifiers, when used in long area applications. The method is quite simple, but it is not very reliable because the accuracy depends on the sensitivity of the measuring block. So the "to the centimeter" measurement becomes a difficult objective to achieve.

Time based location is commonly used in G.P.S [5] and $L O w$ RAnge Navigation (L.O.R.A.N.). This strategy consists of measuring the time delay, on the receiver, between two or more signals from separate transmitters. With that information and the transmitter location identification, it is possible to triangulate the receiver's location in a plain (with two transmitters) or space (with three transmitters) [5]. However, the accuracy of this technique depends on how far the transmitter is away from the receiver, because, the longer the distance the bigger the delay, and therefore the error factor decreases. Fig. 1 shows the time delay between two received signals.

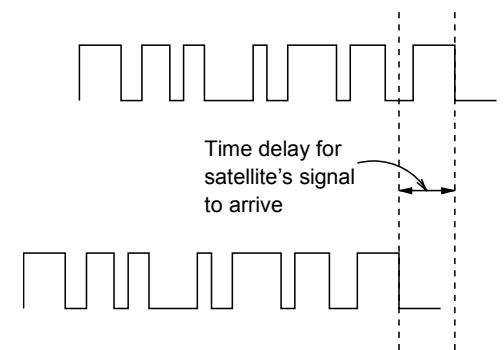

Fig.1 - Time delay between two received signals.

Two other downsides are the atomic clock requirement in the transmitters to keep the signal constant all time, and the periodic synchronism with the others transmitters. These additional systems make this strategy more expensive.

The last approach is the Angle of Reception. With the use of the transmitter's signal strength, it is possible to determine the angle in a plain, on which the signal is stronger with the help of a directional antenna (for e.g. a Yagi antenna). This technique is used, for e.g., in Amateur Radio Direction Finding (A.R.D.F.). There is no need to know the signal strength precise value like in the first strategy. So, the measurement block sensitivity is no longer an issue. But the antenna precision is an important aspect to consider since the antenna aperture (or capture area) must be as smaller as possible. Fig. 2 shows the reception of radio waves on a Yagi-type antenna.

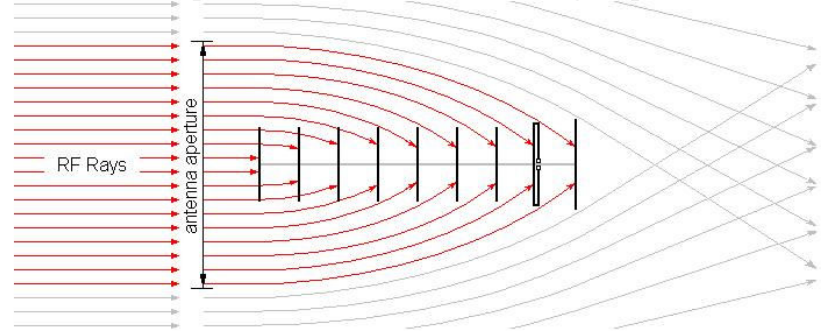

Fig.2 - Reception of radio waves on a Yagi-type antenna.

\section{APPROACH OF THE PROBLEM}

Before stepping up to any kind of electronic design, it is important to review the electromagnetic communication fundamentals. In order to transmit any kind of telecommunication signal the signal frequency (f) is inversely proportional to the wave length $(\lambda)[6]$ :

$$
f=\frac{c}{\lambda}
$$

where $c$ is the speed of the wave, which in the vacuum is the speed of light $\left(3 \times 10^{8} \mathrm{~m} / \mathrm{s}\right)$. Thus, if the signal is transmitted at a very high frequency, the wavelength will be so small that any kind of obstacle will interfere with the transmission. On the other hand, the wavelength is equal to the antenna size (bipolar). Therefore, you can use a small antenna in a portable device. If the frequency value is decreased, the antenna size will increase. The decision is up to de designer.

Let's take the example of football for our application. Using the angle of reception approach doesn't seem to be a good strategy because a ball can move at a high speed and change direction very quickly. To keep up with target movement, it would be necessary for the antenna to sweep the plain, where the ball is inserted, several times in a small interval of time so that the target location refresh rate would be suitable. That fact leaves a choice of the other two strategies. But, as L.O.R.A.N. uses two strategies, a similar approach can be used in this work: Signal strength and Time based. As the first is more efficient at close range, the second is more accurate at distance, and therefore the two combined could perform an excellent solution.

The Signal Strength decreases in a logarithmic way. Using Time based location, it is necessary to measure the delay between the signals received. In a rectangle field, it is possible to use the four corners to implement four transmitters. 
Although it is necessary three points to define a plain, it will be useful to have a fourth transmitter. Then, it would be possible to reduce each signal range, as seen in Fig. 3.

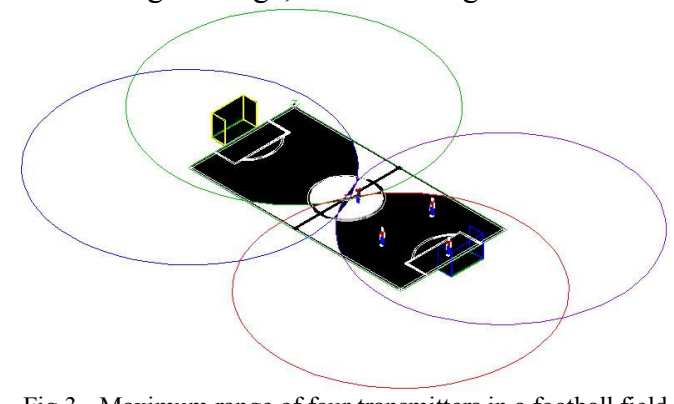

Fig.3 - Maximum range of four transmitters in a football field

The signal from the two corners near a goal, can reach the field centre. A third signal completes the areas where one of the signals cannot reach. Since this strategy is not reliable near a transmitter, the Signal strength should be used. Therefore, we a signal range field for each strategy can be used (Fig. 4).

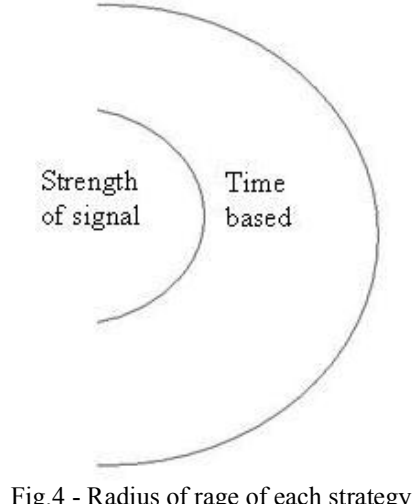

This means it would be necessary to have two systems, making the project more expensive. The solution could be found on the data processing. When a target is near a receiver, this receiver becomes non relevant and the triangulation is made with the three remaining receivers more distant from the transmitter and then the error would be smaller. But it would also mean that the transmitter range would have to be able to reach the most distant receiver, in the worst of cases.

\section{SOLUTIONS}

There are several transmitters/receivers available on the market that can sort out the first problem: telecommunication. When choosing the transmission modules, it is important to check the organization responsible for the local communications (A.N.A.C.O.M. in the case of Portugal) because of restricted frequencies.

The existence of multiple targets is a problem. The solution could be the use of FDMA multiplexing (Frequency Division Multiple Access). Another solution could be the use of shared medium, with the same frequency, but in a time division environment, with TDMA (Time Division Multiple Access) or even CDMA codification (Code Division Multiple Access).
An experience was carried out to analyse the performance of a Radio-location system, measuring the signal strength and how distance (between the transmitter and receiver) affects it. The transmitter was created according to an electronic beacon schematic represented Fig. 5 .

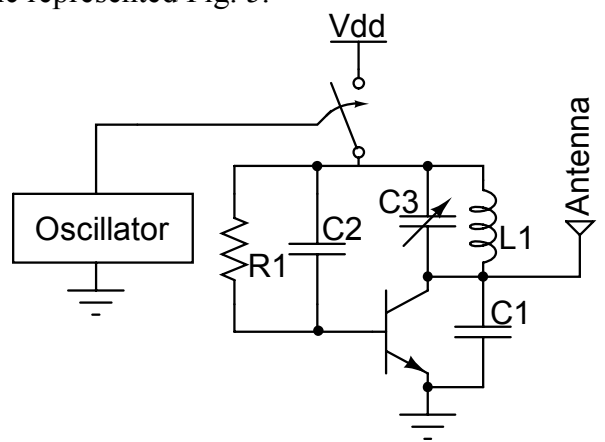

Fig. 5 - Radio Beacon Transmitter of $10 \overline{8} \mathrm{MHz}$ with AM.

This transmitter consists of a block that provides the original signal, which in this case is a simple oscillator (hence the name Beacon) and a transmission block that modulates the original signal in agreement with a Frequency Modulation (FM). The LC circuit (the Coil L1 and Capacitor C3) performs the modulation in $108 \mathrm{MHz}$, where the Coil L1 consists of a copper wire winded two turns and without a ferromagnetic core. Since the Capacitor C3 is a trimmer capacitor, it is possible to regulate the value of this frequency.

The receiver consists of:

- An antenna that transforms the electromagnetic energy emitted by the transmitter;

- $\quad$ An initial filtering block;

- An amplification block and

- $\quad$ An exit filter.

This block diagram is presented in Fig. 6.

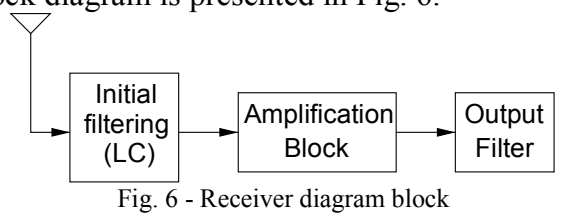

The antenna is the same as used in a common radio receiver and the initial filter is an LC Filter (coil and capacitor) that, in series with the antenna, makes a band pass filter allowing the passage only to signals with frequency:

$$
f=\frac{1}{\sqrt{L \times C}}
$$

The Amplification Block is a common emitter configuration amplifier, using the RF (Radio Frequency) transistor BF199. This configuration is presented on the schematic diagram of Fig. 7. 


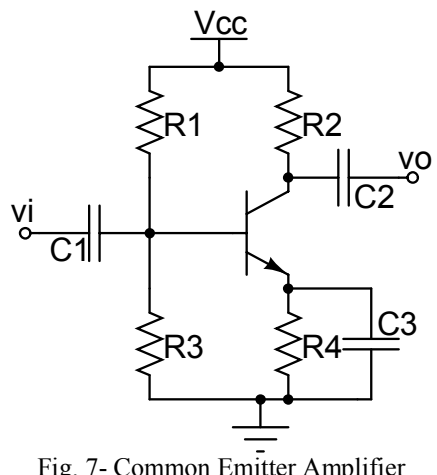

Fig. 7- Common Emitter Amplifier

This experiment was based on the signal strength measured on the receiver, simultaneously changing the distance, but using two receivers with different power gain: one receiver with only one amplification stage and another with three amplification stages. The results obtained, are described in the graphic of Fig. 8. As it is possible to observe, the signal strength decreases as the distance increases. The receiver with one amplification stage isn't capable of receiving the signal originated from the transmitter in an efficient way, to distinguish beyond the noise, a few centimetres from the transmitter. But with three amplification stages, the circuit is capable to reach almost $1.5 \mathrm{~m}$. There exists a discrepancy, in the results, between $95 \mathrm{~cm}$ and $115 \mathrm{~cm}$ but it is believed to due with the fact that the distance is approximately equal to the wave length of the signal, from the antenna, $70 \mathrm{~cm}$.

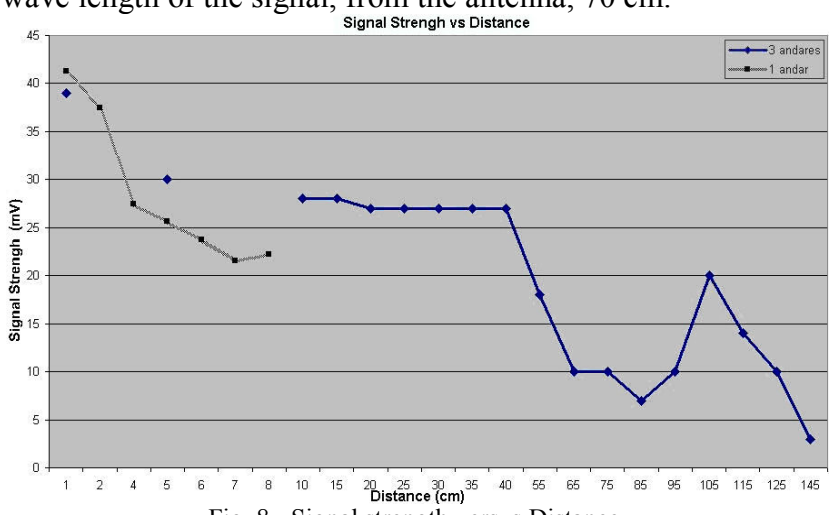

Fig. 8 - Signal strength versus Distance.

This experiment shows that the Signal Strength based strategy is a viable solution for radiolocation.

A second experiment was carried out to verify the Time Based strategy for Radiolocation. This experiment consisted on analysing the impact of distance on the signal arrival time at a receiver.

A $433.92 \mathrm{MHz}$ frequency was used, which belongs to the ISM band (Industrial Security Medical), transmitter with an ASK modulation (Amplitude Shifting Key). This transmitter uses a particular component, to help the modulation process at this frequency: the SR433, a $433,92 \mathrm{MHz}$ resonator which produces a saw shape signal, when $\pm 30 \mathrm{~V}$ is applied.
Combining the SR433 with a small amplification stage, a low power radiofrequency transmitter is obtained. Its schematic diagram is in Fig. 9.

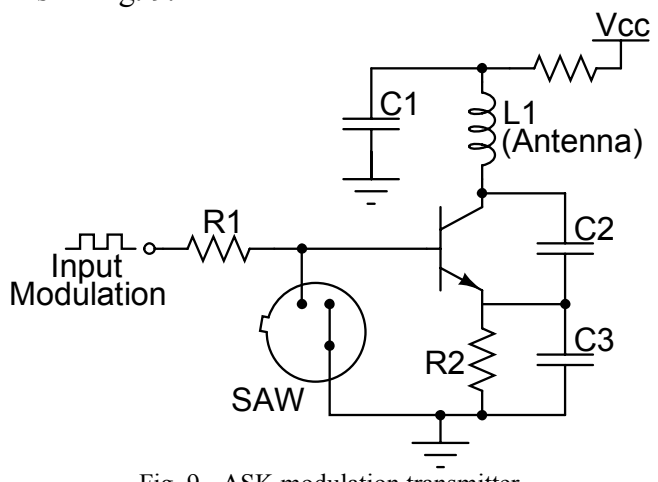

Fig. 9 - ASK modulation transmitter

The previous picture represents the electronic circuit that is integrated in the module TX433N, which requires a $5 \mathrm{~V}$ supply. This module has the capability to transmit digital signals with a maximum output debit of $8 \mathrm{kbps}$. Although it was developed for digital data transitions, the TX433N can be used for analogue based applications. Using this module along with an astable oscillator carefully designed, a beacon transmitter with an original frequency of $1 \mathrm{kHz}$ is obtained.

For the reception, an AC_RX433 module receiver from Aurel was used, for $433,92 \mathrm{MHz}$ with demodulation for ASK. This module is composed by an RF preamplifier, followed by an AM demodulator (Image AM Detector), a low frequency LF (frequency from the original signal) amplifier and finally a peak detector. Fig. 10 shows the block diagram that describes this module.

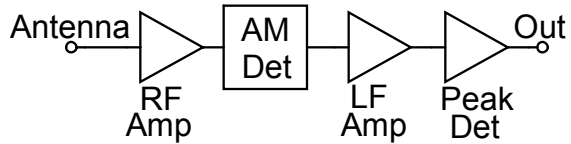

Fig. 10 - Block diagram of the receiver module AC RX433

For this test, two AC RX433 modules from Aurel at 433.92 $\mathrm{MHz}$ were used, as well as an oscilloscope to receive the output waveforms from each receiver and compare them together. The two receivers were placed $9.8 \mathrm{~m}$ apart from each other, and in the centre was placed the oscilloscope. In order for the signals from each receiver to arrive at the same time, four electrical wire were stretched so each one had $4.9 \mathrm{~m}$ precisely, two for each receiver: neutral and phase. The proposed apparatus for this study is pictured in Fig. 11.

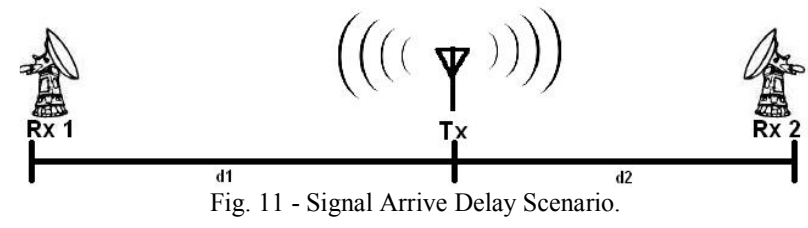

During the preparation of this apparatus, the wires were twisted in order to reduce the noise because the magnetic field created from the phase wire is annulated by the magnetic field from the neutral wire, since the electrical currents in the wires 
are in opposite directions, at all time. It is the same principle used by the Ethernet cables UTP (Unshielded Twisted Pair).

The signals from the two receivers can be seen in the following pictures, one using a $1 \mathrm{~V} /$ div scale and another with $2 \mathrm{~V} /$ div scale (each signal has the same voltage amplitude). The time scale was 20 us/div:

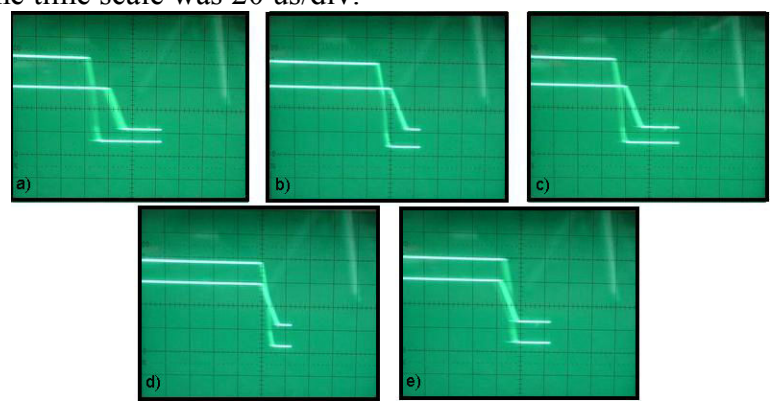

Fig. 11 - Wave form from the two receivers: a) Near the right transmitter; $b$ ) $2.45 \mathrm{~m}$ apart from the right transmitter; c) In the centre; d) $2.45 \mathrm{~m}$ apart from the left transmitter; e) Near the left transmitter.

The five waveforms correspond, respectively, to the scenarios where the transmitter-beacon is:

a) Near the right transmitter;

b) $2.45 \mathrm{~m}$ apart from the right transmitter;

c) In the centre;

d) $2.45 \mathrm{~m}$ apart from the left transmitter;

e) Near the left transmitter.

This experiment results were recorded and it is presented in table 1 .

TABLE 1

RECORDS FROM THE SIGNAL ARRIVE DELAY SCENARIO

\begin{tabular}{|c|c|}
\hline Distance $(\mathrm{m})$ & Signal delay $(\mathrm{ns})$ \\
\hline$\approx 0$ & 80 \\
\hline 2.45 & 60 \\
\hline 4.9 & 50 \\
\hline 7.35 & 20 \\
\hline$\approx 9.8$ & $\approx 0$ \\
\hline
\end{tabular}

The second experiment confirms that, the signal arrive delay changes with the target distance along the line between the two receivers. But these results present a discrepancy from the theoretical presupposition. Accordingly to the laws of Physic, the electromagnetic waves travel at a speed near the speed of light, since the medium where it travels is our atmosphere and not the vacuum. If it is taken into account that the signal travels the atmosphere from the transmitter to the receiver and then through a conductive wire to the oscilloscope, the total distance would double this study distance:

$$
\text { distance }_{\text {total }}=2 \times 9.8 \mathrm{~m}=19.6 \mathrm{~m}
$$

Taking into account that the wave propagation speed is equal to the speed of light, in order to travel $19,6 \mathrm{~m}$, accordingly to Eq.04 the wave would need:

$$
\phi=\frac{l}{c} \Leftrightarrow \phi=\frac{19.6 \mathrm{~m}}{3 \times 10^{8} \mathrm{~m} / \mathrm{s}} \approx 65.33 \mathrm{~ns}
$$

where $\theta$ is the signal delay in seconds, 1 is the distance travelled by the signal and $\mathrm{c}$ is the speed of light which, in vacuum, is $3 \times 10^{8} \mathrm{~m} / \mathrm{s}$.

However, and accordingly to the results, the signal took $80 \mathrm{~ns}$ to travel this distance indicating a large delay along the path. A hypothesis was made in order to explain it: the use of passive components in the receiver modules could explain the signal delay. This passive component could inflict the delay in discussion. In any way, since two similar receiver modules were used, it is possible to say that this problem affects the two modules on the same way. So even with this difference between the theoretical and practical results, the relation Signal Delay versus Distance is here proven.

\section{CONCLUSIONS}

A practical implementation for a radiolocation system is possible with a minimal economical and time effort. It is up to the system designer to analyse whether the technology here presented is advantageous taking into account the needs of the project.

Granted that much study is needed to prefect this system, but further work will eventually bring considerable results for a system capable of location using radiofrequency.

\section{REFERENCES}

[1] http://www.futureofflight.org, Aviation Center \& Boeing Tour. Viewed at 2006/11

[2] http://www.boeing.com/companyoffices/aboutus/tours/, Boeing: Aviation Center \& Boeing Tour. Viewed at 2006/11

[3] Mota, Sara Piteira. FIFA aprova bola com 'chip' em jogos amadores. Diário Económico. (09 Mar. 2007);

[4] http://www.hawkeyeinnovations.co.uk/?page_id=1009, Hawk-Eye Innovations. Consultado em Agosto 2007

[5] Kaplan, Elliott D. (ed.) Understanding GPS: principles and applications. Artech House, Norwood (1996);

[6] Vaz, Emanuel Eduardo Pires. Antenas - Teoria e Prática. Edições Lopes da Silva, Porto (1987); 